\title{
TRANSFER OF TRAINING: IMPROVING THE EFFECTIVENESS OF EMPLOYEE TRAINING IN NEPAL
}

\author{
Bhawani Shankar Subedi*
}

Abstract

This paper presents a summary, conclusions and recommendations

of a recent doctoral study titled 'Transfer of Training: Improving the Effectiveness of Employee Training in Nepal'.

The purpose of this study was to examine the extent of transfer and to identify factors influencing it in the context of civil and corporate sector organizations of Nepal. This cross sectional descriptive research included stratified random samples of organizations from four development regions of Nepal. Data were collected from 299 cases (56 managers, 78 supervisors and 165 employees, who received training within the last twelve months) using a mail survey, on-site survey, personal interviews,

focus group discussions, training evaluation models, and observations for the verification of workplace evidences of transfer or non-transfer of the training.
Most organizations in Nepal invest in training. Effectiveness of training was found constrained due to limited transfer of learning from the training environment to the workplace environment. Training stakeholders such as managers, supervisors, employees, training designers and providers were found working in isolation instead of working together for a common purpose. In most cases, not enough attention was given to what happened before, during, and after completion of training. Follow-up and tracer studies were generally not done or the recommendations not used. Consequently, training- job relevance and the extent of transfer were found limited by factors related to the trainees, training design and the workplace environment. Findings could be useful for the review of human resource development policies and practices leading to an increased extent of transfer of training in the future.

\section{Overview}

This paper presents a summary of the study on transfer of training, summary of its major findings, conclusions and recommendations. Based on the results and discussions, summary of major findings are presented in sequence according to the themes of the research questions addressed in this study. Conclusions drawn from the findings also correspond to the research questions. Recommendations offered to the training stakeholders and for further research are based on hypothesized and non-hypothesized findings of the study. Suggestions for improving the effectiveness of employee training are offered. The article concludes with recommendations for future research.

* Training Institute for Technical Instruction (TITI). 


\section{Summary of the Study}

This study belonged to the area of training and development with focus on the issue of transfer of training. Transfer of Training was defined as the extent to which the knowledge, skills and attitudes gained from the training environment are retained and used in the workplace environment.

\section{The Research Problem}

Employee training effectiveness remained often questioned. However, the extent of transfer and factors influencing the process and outcome of transfer of training were not studied in the past in Nepal. An interest and the need to fill up that gap prompted this study.

Previous studies that contributed to our knowledge and insight in the area of training and development, often researched effects of factors in isolation, and in fact, in the contexts other than those that typically prevail in the organizations of Nepal.

Transfer of training, though it is often the only valid measure of training effectiveness (Holton and Bates, 1998), did not receive adequate attention in the context of Nepal (Subedi, 2002).

Transfer of training (or lack of it) was identified as a complex process and dependent upon the intent or motivation of the learner (trainee related factors), the organizational environment and culture including supervisory support (organizational or workplace related factors), and the instructional design, delivery features as well as job relevance of the training program (training related factors).

Training effectiveness in Nepal was found arguably constrained due to inadequate transfer of learning from the training environment to the workplace environment. Training stakeholders such as managers, supervisors, employees, training providers and designers continued working in isolation instead of working together for a common purpose.

\section{Purpose of the Study}

The purpose of this study was to examine the extent of transfer of training and to identify factors influencing it. This purpose originated from the need to improve the effectiveness of employee training in the context of civil and corporate sector organizations of Nepal.

In congruence with the purpose this research examined the extent of transfer of training, identified and ranked factors that influenced the effectiveness of employee training in Nepal. Additionally, this study explored how managers, supervisors and employees perceived transfer of training in their own organizations and how their actions influenced employee training effectiveness.

This study also sought to contribute to the expansion of the current body of knowledge about transfer of training, especially in contexts other than those that prevail in the modern workplaces of the western culture.

\section{Research Questions}

To meet the purpose of the study, the following research questions were addressed:

1. To what extent is transfer of training occurring in Nepali organizations?

2. What are the common factors that influence transfer of training in the organizations?

3. How close are the estimates of managers, supervisors and employees to each other and to the obtained value of transfer of training?

4. Are the western models and instruments appropriate for interpreting transfer of training in the context of Nepali organizations?

5. In the organizational culture of Nepal, what are the factors and beliefs influencing transfer of training? 


\section{Setting and Sample}

The target population of this study included all managers, supervisors and employees of the 'civil' and 'corporate' sector organizations of Nepal. From a total of 350 organizations having 50 or more employees, a stratified random sample of 50 organizations, covering two sectors and four development regions, was selected as the initial sample. A total number of 299 cases were included by means of six different research instruments used to collect on-site data for this study.

\section{Outcome of the Study}

This study investigated the estimated ranges and calculated rates of the extents to which the knowledge, skills and attitudes gained from the training were retained and applied on the job. Factors influencing transfer of training were identified and ranked in terms of intensity. Comparisons were made for the extents of transfer of training between civil and corporate organizations and among the managers, supervisors and employees.

Additionally, factors and beliefs about transfer of training in the organizational culture were analyzed. Western models and instruments interpreting transfer of training were reviewed and their appropriateness for use in the context of Nepal was assessed.

Since this is the first doctoral study in the area of transfer of training in Nepal, findings and recommendations made in this study are expected to be useful for the review of human resource development policies and practices leading to a greater extent of transfer of training in the future. Practically, all training stakeholders including employers, sponsors, training providers, designers and trainees can benefit from the outcome of this study.

\section{Summary of Major Findings}

This study was designed to examine the extent of transfer of training, and to identify factors influencing the process and outcome of employee training effectiveness in the context of civil and corporate sector organizations of Nepal. Using a conceptual framework of 'organizational or workplace related factors', 'training design and delivery related factors' and 'trainee related factors', transfer of training was examined and factors influencing it were identified and ranked. Extent of transfer of training was assessed. Estimated ranges and calculated rates of transfer were compared. Some of the frequently used western models and instruments on transfer of training were reviewed and their appropriateness in the context of Nepal was assessed. Likewise, cultural factors or beliefs about transfer of training in the organizational culture were identified and assessed.

Data collected for this study were classified according to the research questions. Findings and discussion of the data sought to answer those research questions. Chapters four through eight presented findings and discussion of the data and results corresponding to the following themes of the research questions:

\section{Research Question 1: The Extent of Transfer of Training}

1. The overall rate of the extent of transfer of training in the organizations was found 56.89 percent on the average.

2. Results of this study found that corporate (non-government) sector organizations in Nepal have 69.33 percent transfer of training on the average.

3. Average rate of the extent of transfer of training in the civil (government) sector organizations was found 47.57 percent.

4. The 21.76 percent higher rate of transfer of training in the case of corporate sector indicated that employee training effectiveness is higher in the corporate sector organizations than in the civil sector. The following factors were found operating more positively in the corporate than in the 
civil sector that possibly led to the higher extent of transfer of training:

5. Provided opportunities to practice new skills on the job,

6. Helped employees with resources and time to perform new skills,

7. Encouraged employees to seek training options, and

8. Required supervisors to work more closely with the employees.

9. Training-job irrelevance, pre-training knowledge/skill and incomprehension or inadequate learning of the objectives and tasks included in the training courses were factors contributing to non-transfer.

10. Number of objectives contained in the course and the number of participants in the training did not seem to have an impact on the extent of transfer of training.

11. Training courses with relatively higher pretraining knowledge/skill rates were only partially challenging for the participants.

12. Observations for the workplace evidences of transfer indicated an agreement between the employees and their supervisors about the objective(s) that transferred to the workplace.

\section{Research Question 2: Factors Influencing Transfer of Training}

1. Results indicated that employees were expected to use new skills and knowledge after training but they were not rewarded or promoted for using the learning from the training.

2. Supervisors supported the use of new knowledge and skills after the training but the organizational and management support to apply new skills was reported inadequate.

3. Supervisors and employees of both the civil and corporate sector believed that their 'organizations distinguish between good and bad performance' whereas more than average civil sector managers do not 'assess performance based on the use of new knowledge and skills'.

4. Supervisors as well as employees of both the sectors believed that their 'organizations select the right people for the training' however, there is a tendency of not 'involving supervisors and employees in planning their own training', more so in the case of civil sector organizations.

5. Over the issue of 'providing resources and time to apply new learning on the job' most managers of the civil organizations were found indifferent. That is, they neither supported nor hindered. Managers of the corporate sector took some initiation about providing resources and time to apply new skills on the job.

6. Factors related to training design or delivery identified as positively influencing transfer of training included job-related design of training courses, building confidence and willingness to change after training, training based on job or performance requirements and quality of training for the employees.

7. Findings showed that 'staff gained confidence and willingness to change after training', but 'training opportunities for the staff who perform better' were not generally available.

8. 'Follow up and feedback after training' appeared to be a relatively weak aspect in facilitating transfer of training.

9. The 'trainee' himself/herself was identified as the most important factor as a facilitator or barrier to transfer of training.

10. In comparison with other factors such as 'quality of training' and 'workplace support', 'trainee characteristics' suchaspersonalability, self-efficacy, motivation, commitment and willingness to seek opportunity to perform 
better are factors that seemed to be more powerful in influencing transfer of training.

11. 'Quality of training' and 'workplace support' were identified other important factors influencing transfer of training.

12. Employees who exhibited 'increased selfconfidence and ability after training' and 'sought feedback and opportunity to perform better' seemed to positively influence transfer of training in their organizations.

13. Employees who actively participated in training, shared improvements with others and sought feedback were likely to positively influence transfer of training.

14. Effective transfer of training required action plans, supervisor support and practice opportunities in the workplace.

15. It was found that employee performance was not assessed based on 'efforts to apply new skills on the job'.

16. Employees were left alone without support when they attempted to use the new knowledge and skills in the workplace.

\section{Research Question 3: Estimates of Transfer of Training}

1. Majority of the managers (53.8 percent) estimated that the rate of transfer of training in their organizations was between $41-60$ percent. They were found better estimators of transfer compared to the overall extent $(56.89 \%)$.

2. Findings indicated that managers of the civil sector organizations tend to overestimate the extent of transfer of training occurring in their organizations.

3. Average extent of transfer of training (56.89 $\%$ ) in both types of organizations indicated that managers of the corporate tend to estimate less than actual (69.33\%).

4. Supervisors and employees of both the sectors were found widely scattered over their range of estimates as compared to the overall estimate $(41-60 \%)$.

5. Test of significance for the differences in the estimates of transfer of training between supervisors and employees, and between civil and corporate sectors did not indicate a statistically significant difference.

Research Question 4: Appropriateness of Western Models and Instruments

1. Most of the western models and instruments found in the literature on transfer of training were found derived from industrial psychology and organizational behavior.

2. 'Transfer of Training' reflected Kirkpatrick's third level in the evaluation model. This was a measure of the extent to which participants change their on-the-job behavior because of training.

3. Kirkpatrick's four-stage model could not be a readymade capsule for evaluating transfer of training in the organizations without having a set of pre-defined criteria for success at each level. It is because much training was delivered without a plan for measuring the transfer of training.

4. Role-time model (Broad and Newstrom, 1992) was found appropriate in case of training activities or courses that had no formal structure or written objectives but still were reported as 'successful' or 'useful' by the participants and their supervisors. It focuses on stakeholder approach to training.

5. Transfer Of Training Evaluation Model (TOTEM, 1995) was identified a tough model because it excluded pre-training knowledge/skill as well as training-job irrelevance and incomprehension rates of training courses from the rates of transfer of training.

6. This model (TOTEM, 1995) could be used and interpreted effectively for any course 
with identified competencies written in the form of objectives or tasks.

7. The learning Transfer Systems Inventory (LTSI) model (Holton and Bates, 1998) was found useful because it could be instrumental as an advance organizer for the instrument constructs and for interpretation of the data on factors influencing transfer of training in the context of organizations in Nepal.

\section{Research Question 5: Cultural Factors and Beliefs Influencing Transfer of Training}

1. Managers, supervisors and employees all expressed their needs to be clear about training-job relevance, trainee selection, training policy and plans, workplace support, perceived value of training, training design, non-training problems, posttraining support and purpose of training for improving the effectiveness of employee training in Nepal.

2. Results showed that managers, supervisors and employees perceived the valuable of training for performance improvement $(60.78 \%)$, for achieving organizational goals $(54.90 \%)$, and as a means of rewarding good performers (52.94\%).

3. Likewise, supervisors and employees believed that the reason for sending people to training was for achieving organizational goals (94\%), performance improvement $(87.33 \%)$, and for rewarding good performers $(53.33 \%)$.

4. The $78.43 \%$ (40) of the persons interviewed (51), and $86.67 \%$ (130) of the persons surveyed identified 'organizational needs' as the most important criteria used in employee selection for training.

5. 'External pressure' (political or organizational pressure from the higher rank, 'sourceforce' or 'bhansoon') $7.84 \%$, and 'internal bias' ('afnoo manche' and 'chakadi') also $7.84 \%$, were identified as the other factors influencing selection of employees for training.
6. Managers, supervisors and employees identified 'transfer of knowledge, skills and attitudes from the training to the job' $(70.58 \%)$, 'gain in knowledge, skills and attitudes' (50.98\%), and 'achieving better organizational results' $(41.17 \%)$ as the most important criteria for any training to be called perfect.

7. The concept of 'return on investment' was not recognized as an important criterion for a training to be perfect (only $7.84 \%$ (4) of the cases reported this as a criterion).

8. Suggestions to improve the effectiveness of employee training in Nepal included the following themes derived from a total of one hundred and thirty-five statements of suggestions collected from managers, supervisors and employees who received training within one year:

a. Provide only job-related and need-based training. Similarly, job after training must be training related $(62.74 \%)$.

b. Avoid biasness in staff selection for training to ensure right person for the right training $(50.98 \%)$.

c. Work out a well defined HRD policy and transparent plans to make staff training effective $(47.06 \%)$.

d. Require employees to improve performance after training. Create a supportive workplace environment in the organization (31.37\%).

e. Discourage the tendency to take training as a means of financial gains or relief from work (25.5\%).

f. Design and deliver training that is practical and responsive to real workplace problems (17.64\%).

g. Do not overuse training as the solution to all kinds of workplace problems (13.72\%). 
h. Conduct post-training follow-up to help to increase training effectiveness (7.84\%).

i. Use training as a means to facilitate better job performance in the context of organizational or job changes (7.84\%).

\section{Conclusions}

Five research questions and six instruments for data collection guided this researcher throughout the study. Based on the discussion of the results and findings, the following conclusions have been drawn and grouped according to the themes of the research questions addressed in this study:

\section{The Extent of Transfer of Training}

1. Determining the extent of transfer of training is an objective measure of training effectiveness evaluation in terms of quantifying retention and application of the knowledge, skills and attitudes from the training environment to the workplace environment.

2. The actual rate of transfer of training proportionately depends upon the extent of pre-training knowledge, training-job irrelevance and rate of incomprehension from any course or program. In other words, increasing the rate of transfer of training requires a proportionate decrease in the rates of other variables.

3. The overall rate of the extent of employee training effectiveness currently occurring in the organizations of Nepal (56.89\%) remained inconclusive due to the nonexistence of any norm or standard to compare at present in Nepal.

4. The extent of transfer of training provided to the employees of the organizations belonging to the corporate sector (non-government) is higher than that of civil sector (government) organizations in Nepal.

\section{Factors Influencing Transfer of Training}

1. 'Organizational or workplace related factors', 'training design and delivery related factors' and 'trainee related factors' provide a useful classification of factors influencing training effectiveness in terms of transfer of training.

2. Effectiveness of any training in terms of transfer of knowledge, skills and attitudes from the training environment to the workplace environment depends upon the 'trainee' himself or herself. This conclusion is drawn from the finding that 'trainee related factors' such as pre-training motivation, selfefficacy, commitment to apply and personal abilities were identified as the most critical factors influencing transfer of training. In other words, lack of confidence, absence of motivation and personal readiness on part of the trained employee would lead to low transfer or negative transfer of training.

3. Major factors and conditions positively influencing the extent of transfer of training include:

a. Relevance between training and job.

b. Clear expectations from the organizations for the application of knowledge and skills from the training to the job of the employees.

c. Training designed and conducted based on identified needs.

d. Initiation for change and adequate support from the employer and supervisor to the employees who received training.

4. Major factors and conditions negatively influencing transfer of training include:

a. Where 'efforts to apply new knowledge and skills' do not constitute performance appraisal criteria.

b. Where there is inadequate follow up support and feedback to the trainees after the training. 
c. When there is a 'feeling of no reward or promotion' for using the learning from the training.

\section{Estimates of Transfer of Training}

1. Executives and managers are better estimators of transfer of training than supervisors and employees. Majority of the managers' estimate of transfer of training was found within the range of actual transfer of training occurring in their organizations.

2. Managers of the corporate sector tend to estimate less than the actual transfer of training occurring in their organizations. Likewise, managers of the civil sector have the tendency to estimate higher than the actual transfer of training occurring in their organizations.

3. Supervisors and employees of both the sectors are closer to each other in their estimates but they exhibit a more widely scattered trend in their transfer of training estimates.

4. Factors and conditions leading to a higher extent of transfer of training in the case of corporate sector include better opportunity to practice, resources and time, seeking training options, supervisor support, increased training-job relevance, tendency to seek feedback, action plans and motivation to learn and apply.

\section{Appropriateness of Western Models and Instruments}

1. Because much training is delivered without a plan for measuring the transfer of training, the 'four-stage training evaluation model' (Kirkpatrick, 1959) cannot be used as a readymade capsule for evaluating transfer of training in the organizations without having a set of pre-defined criteria for success at each level. However, this model was found easy to understand and interpret employee training effectiveness in the context of Nepal.

2. The 'role-time model of transfer of training' (Broad and Newstrom, 1992) is a useful classification system on three fronts: to understand the different dimensions of a transfer partnership, to identify the transfer strategies, and to identify the barriers influencing transfer of training.

3. Transfer of Training Evaluation Model (TOTEM, 1995) is an objective model to derive training transfer rates in figures. Outcome depends upon the honest responses of the participants.

4. The Learning Transfer Systems Inventory (LTSI) model (Holton and Bates, 1998) is useful as an advance organizer for the instrument constructs and interpretation of the data on factors influencing transfer of training in the context of Nepal, too.

\section{Cultural Factors and Beliefs Influencing Transfer of}

Training

1. Organizational culture and beliefs held by the managers, supervisors and employees about training and development are likely to influence the process as well as outcome of employee training in Nepal.

2. The stakeholders do not yet recognize the concept of 'return on investment' as a critical success factor in the context of employee training in Nepal.

3. Training as a 'means of rewarding good performers' is identified as an emerging basis of employee selection for training in both the civil and corporate sector organizations.

4. Value of training as an aid for 'promotion to higher positions' among the civil sector employees is overriding the importance of training for better performance and better organizational results.

5. In spite of the awareness about the value of training as the 'need for better organizational results' and 'performance improvement', training opportunities are not adequate for those employees who exhibit a tendency to 
perform better in their current as well as future job.

6. Current practices of employee selection for training indicate a situation not much in favor of utilizing training for better performance and better organizational results.

7. Since 'training and job relevance' is identified as the most important factor in the case of employee training in Nepal, objectives and content areas of training courses with higher 'training-job irrelevance' rates need revision or else exclusion from the courses to improve the effectiveness of employee training in Nepal.

\section{Recommendations}

On the basis of results and discussions of the findings, and in congruence with the conclusions drawn from of this study on transfer of training, the following two types of recommendations are offered:

1. Recommendations offered to the training stakeholders that includes employers, employees, managers, supervisors, sponsors, training designers and training providers.

2. Recommendations for further research that includes the need for additional research in the area of transfer of training and situations where this research could be replicated.

\section{Recommendations Offered to the Training Stakeholders}

1. Improving the effectiveness of employee training in Nepal requires increasing the extent of transfer of training. Training stakeholders should make efforts to proportionately reduce training-job irrelevance, assess pre-training knowledge and reduce incomprehension rates.

2. Right training for the right person, appropriate training needs assessment and effective design and delivery of the training could be instrumental in achieving higher rates of transfer of training.

3. Training-job relevance of the training courses for employees needs improvement. A thorough review in the beginning and a periodic update of the contents and objectives, involving managers, supervisors and employees, is recommended. Focus group interviews and discussions are recommended to facilitate this process in all organizations where there is a provision of employee training.

4. The reportedly high pre-training knowledge rates on the one hand, and inadequate confidence among employees on the other hand, illustrated hindrances in the application of the knowledge and skills from the training to the job. Pre-training assessment of knowledge and skills, and a participatory way of formulating consented training goals are recommended for increasing the effectiveness of employee training.

5. Based on the observation that employees exhibited different levels of confidence in learning and application, it is recommended that future training should be offered to homogeneous groups for increasing the extent of transfer of training for better results in the organizations.

6. Since the reported indifference on the part of the managers was found to be an important cause for the non-attempt to apply new skills on the job, this study recommends an annual orientation or conference about employee training plans in the organizations. This annual event could focus on the purpose, expected learning outcome, contents, and objectives of the training activities foreseen for the whole or part of the year as appropriate.

7. It is also recommended to require every employee to debrief and communicate 
action plan for the application of knowledge and skills gained from any training.

8. It is recommended that training stakeholders (managers, supervisors, employees, sponsors, and training providers) should establish mutually agreed upon criteria for training success, and expected rates of transfer for various types of training courses.

9. Managers, supervisors and employees of the organizations should be invited to share their expectations and organizational needs for training and development.

10. Lack of resources and opportunity to perform skills from the training back at the workplaces was found a significant barrier to effective transfer of training. Action planning for the application of the knowledge and skills from the training to the job, also considering the participants' organizational constraints and opportunities, could help increase the rates of transfer of training. Such action plans should include what alternatives are available and how cognition works at different situations for the use of the knowledge and skills on the job.

\section{Recommendations for Further Research}

1. This study identified and ranked factors and conditions that influence the extent of transfer of training. Transfer of training is apparently influenced by trainee related factors, workplace related factors and training related factors. Further research is recommended to explore which of the factors affecting transfer of training can be manipulated or changed, and how positive transfer can be increased.

2. Normative standard for the acceptable extent of transfer of training was nonexistent for reference. Although literature on training and development suggests $40 \%$ as the minimum acceptable rate of transfer, this standard could be different for different types of coursers- technical or softskills. Further research is recommended to determine 'normative standards' to compare and evaluate effectiveness of any training in terms of the extent of transfer.

3. Further research is recommended to include larger sample data to verify the result of this study that the corporate sector has a higher extent of transfer of training than the civil sector organizations in Nepal.

4. When this research is replicated-use a larger sample data including military, police, diplomatic missions and multiple national companies, reduce the number of research instruments from six to two and focus on verification to consolidate the results,

5. collect longitudinal data from the same organizations covering a period of two to three years instead of making it a crosssectional, and focus on the effect of particular training courses provided to different types of participants (such as male-female, civilcorporate, newly appointed and experienced participants).

\section{References}

Baldwin, T. T. \& Ford, J. K. (1988). Transfer of training: A review and directions for future research. Personnel Psychology, 41; pp. 63-105.

Bandura, A. (1997). Self-efficacy: The exercise of control. New York: W. Freeman.

Best, W. J., \& Kahn, V. J. (1998). Research in education. Singapore: Prentice Hall.

Bigge, M. L. \& Shermis, S. S. (5th edition) (1992). Learning theories for teachers. New York: Harper Collins.

Bramley, P. (1996). Evaluating training effectiveness: London: McGraw Hill International.

Broad L. \& Newstrom W. J. (1992) Transfer of Training: New York: W. Freeman.

Ellis, H. C. (1965). The transfer of learning. New York: The Macmillan Press.

Foxon, M. (1993). A process approach to the transfer of training Part 1: The impact of motivation and supervisor support on transfer maintenance: 
Australian Journal of Educational Technology, 9(2).

Foxon, M. (1994). A process approach to the transfer of training Part 2: Using action planning to facilitate the transfer of training: Australian Journal of Educational Technology, 10(1).

Gist, M. E., Bavetta, A. G., Stevens, C. K. (Spring 1990). Transfer training methods: its influence on skill generalization, skill repetition, and performance level. Personnel Psychology. (43)3.

Kirkpatric, D. (1996). Great ideas revisited. Vol. 50 (pp.5465) Training and Development. 01-01-1996.

Ford, J. K. (1990). Understanding training transfer: the water remains murky. In Human Resource Development Quarterly; 1, (pp. 225-29).

Misko, J. (1999). The transfer of knowledge and skill to different contexts- An empirical perspective. Leabrook, SA, Australia: NCVER.

Noe, R. A. \& Schmitt, N. (1986). The influence of trainee attitudes on training effectiveness: Test of a model. Personnel Psychology. Vol. 39. (pp.497-523).

Parry, B. S. (May 1991). Linking training to the business plan. In Training and Development, 45(5), pp. $32-54$.

Quinones, M. A., Ford, J. K., Sego, D. J., \& Smith, E. M. (1995). The effects of individual and transfer environment characteristics on the opportunity to perform trained tasks. Training Research Journal, vol.1 (pp.29-48).

Rothwell, W. J. \& Sredl, H. J. (Eds) (1992) The ASTD reference guide to professional human resource development roles and competencies. (2nd edition). ASTD: HRD Press Inc.

Salas, E. \& Cannon-Bowers, J. A. (2001). The science of training: A decade of progress. Annual Review of Psychology. 52, 471-99.
Subedi, B. S. (2002). Instructor training effectiveness: Measuring post-training retention and application of instructional skills on the job. Unpublished M. Phil. thesis, Kathmandu University, School of Education; Kathmandu.

Subedi, B. S. (2002). Employee Training Effectiveness Survey. Unpublished Research Seminar Paper, Kathmandu University, School of Education; Kathmandu.

Tannenbaum, S. L. \& Yukl G. (1992). Training and development in work organizations. Annual Review of Psychology, (43): 399-441.

Taylor, M. C. (Spring, 2000). Transfer of learning in workplace literacy programs. Adult basic education; Vol. 10, Issue. 1.

Tuijnman, A. C. (Ed.). (1996). International encyclopedia of adult education and training (2nd edition). London: Pergamon.

Viviene E. C. \& Cathlin, M. (Eds.) (2000). Transfer of learning in professional and vocational education. London: Routledge.

Vosburg, W. J. (2000). Factors affecting transfer of training from the classroom to the worksite. Unpublished dissertation for the degree of doctor of education. UMI dissertation services: A Bell \& Howell company, Michigan, USA.

TOTEM (1995) Transfer of Training Evaluation Model, Waste Isolation Division, New Mexico: Author.

Wexley, K. N. \& Latham, G. P. (1991). Developing and training human resources in organizations (2nd ed.) New York: Harper-Collins.

Yamnil, S. \& McLean, G. N. (2001). Theories supporting transfer of training. Human Resource Development Quarterly, 12(2).

Zielinski, D. (1996). Evaluating training's impact. Minneapolis: Lakewood Publications. 